\title{
Arteriosclerosis in the Influx and Intravisceral Arteries of the Liver, Kidney and Lung of WHHL Rabbits
}

\author{
Ryozo KAMIMURA1), Syusaku SUZUKI1), Kenkichi MIYAHARA2), and Masashi SHIOMI3)
}

\author{
1)Institute of Laboratory Animal Sciences, Faculty of Medicine, Kagoshima University, 8-35-1 \\ Sakuragaoka, Kagoshima 890-8520, 2)Sinkyo Hospital, 3-41-1 Usuki, Kagoshima 890-0073, \\ and ${ }^{3}$ Institute for Experimental Animals, Kobe University School of Medicine, 7-5-1 \\ Kusunoki-cho, Chuo-ku, Kobe 650-0017, Japan
}

\begin{abstract}
We performed a histopathological investigation on arteriosclerotic development in the influx and intravisceral arteries of the liver, kidney and lung of male WHHL rabbits. In the influx arteries of these organs, we observed severe atherosclerotic vascular lesions with high-grade luminal stenosis. In the intravisceral arteries of the liver and kidney, no arteriosclerotic lesions were observed. However, in the intrapulmonary arteries, we recognized severe atherosclerotic vascular changes with high-grade stenosis or total obstruction of the lumen in some middle to large sized pulmonary arteries. These observations indicate that the development of arteriosclerosis in parenchymatous organs differs, and that some organs are predisposed to arteriosclerosis formation.
\end{abstract}

Key words: arteriosclerosis, organ-specificity, WHHL rabbit

The Watanabe heritable hyperlipidemic (WHHL) rabbit, which spontaneously forms atherosclerosis, has been regarded as an animal model for human atherosclerosis $[1,6,10]$, and familial hypercholesterolemia especially $[2,3,15]$. There have been many reports on sclerotic lesions of the coronary artery $[7,11,13,16]$ and aorta $[4,5,8,9]$ until now, but there are no reports on the arterial lesions in the influx and intravisceral arteries of the parenchymatous organs, except for the heart and brain [12], in the WHHL rabbit. In the present study, we investigated histopathologically the arteriosclerotic changes in the influx and intravisceral arteries of the liver, kidney and lung, and clarified the degree and progress level of arteriosclerosis in these parenchymatous organs.
Five male two- year-old WHHL rabbits weighing $3.2-3.5 \mathrm{~kg}$ were used in this study. The rabbits were caged in an automatically conditioned room (temperature: $22 \pm 2^{\circ} \mathrm{C}$; humidity: $55 \pm 10 \%$; air change: 10 times/hr; $12 \mathrm{hr}$ light/12 hr dark cycle) and were fed a standard rabbit chow. Water was provided ad libitum. They were euthanatized with an over-dose of sodiumpentobarbital and the liver, kidney and lung were removed immediately including all the influx arteries. Fixation of the organs was performed by immersing in $10 \%$ buffered formalin. After paraffin embedding, 5$\mu \mathrm{m}$-thick sections were stained with hematoxylin-eosin (H E) or Van Gieson. In the liver, the celiac trunk bifurcation, common hepatic artery, proper hepatic artery of the porta hepatis, and interlobular artery were

(Received 14 June 2000 / Accepted 14 February 2001)

Address corresponding: R. Kamimura, Institute of Laboratory Animal Sciences, Faculty of Medicine, Kagoshima University, 8-35-1 Sakuragaoka, Kagoshima 890-8520, Japan 

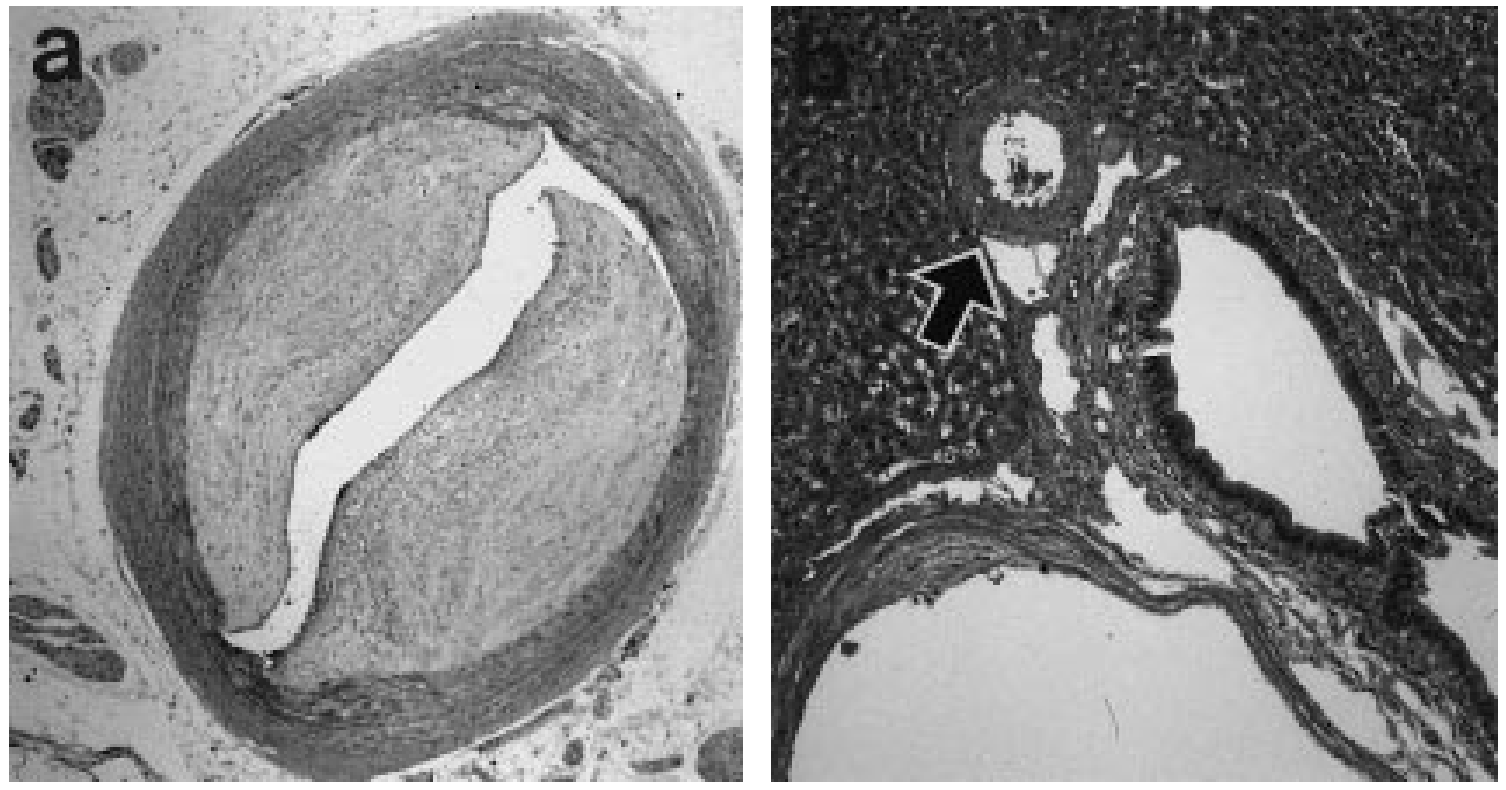

Fig. 1. The influx and intravisceral arteries of the liver. (a): Severe luminal stenosis was seen in a common hepatic artery $(\times$ 25) (stenotic rate: $85 \%$ ). (b): No sclerotic change was observed in an interlobular artery $(\times 25)$.

histologically examined. In the kidney, the target vessels of the examination were the bifurcation of the renal artery, renal artery in the hilum of kidney, arteriae interlobulares renis, and arteriae interlobulares. In the lung, the pulmonary artery trunk, pulmonary artery of the hilus, and intrapulmonary artery were investigated. The rate of vascular lumen stenosis was calculated on a computer using an image analysis software (NIH Image Ver.1.56). The stenosis rate was defined as follows:

Stenosis rate $(\%)=($ area of intimal thickening/area of native lumen of the artery) $\times 100$

The present study was carried out after receiving permission from the Committee of Animal Experimentation, Faculty of Medicine, Kagoshima University.

[Liver] The influx arteries: In the celiac trunk bifurcation, hepatic artery and proper hepatic artery, advanced arteriosclerotic lesions with severe luminal stenosis were recognized and the stenotic rates were 50-90\% (Fig. 1.a). The severity of lumen stenosis was advanced to close to the porta hepatis. Fibrous connective tissue and wide hyalinization were characteristics of the lesions. The intrahepatic arteries: Though a slight intimal proliferation was partially recognized in the proper hepatic artery right after the porta hepatis, arteriosclerotic lesions were not observed in the interlobular artery (Fig. 1.b). [Kid- ney] The influx arteries: The renal artery showed severe stenosis and the stenotic rates of the base and hilus renalis were $60-80 \%$ (Fig. 2.a). The intrarenal arteries: In some interlobar arteries right after the hilum, a slight intimal proliferation composed of foam cells could be recognized (Fig. 2.b), but no scleromatous lesions were observed in any other vessels. [Lung] The influx arteries: Severe atherosclerotic lesions were observed in the pulmonary arterial trunk. Complicated lesions with cholesterol clefts and fibrous cap were recognized (Fig. 3.a). The intrapulmonary blood vessels: In some large sized intrapulmonary arteries, severe vascular lumen stenosis due to the intimal proliferation of foam cells and fibrous connective tissue were frequently recognized. Stenotic rates of the vessels reached $80-90 \%$ (Fig. 3.b) and a vessel in which the vascular lumen was obstructed entirely was observed. Furthermore, vascular lumen stenosis was recognized in some middle sized intrapulmonary arteries, too. To date, there have been no reports on the lesions in the influx and intravisceral arteries of parenchymatous organs except for the heart $[7,11,13$, 16] and brain [12] in WHHL rabbits. In the present study, we investigated arteriosclerosis in the lung, liver and kidney. In the influx arteries of the liver, kidney and lung, high-grade arteriosclerotic lesions were observed. The representative lesions of the pulmonary 

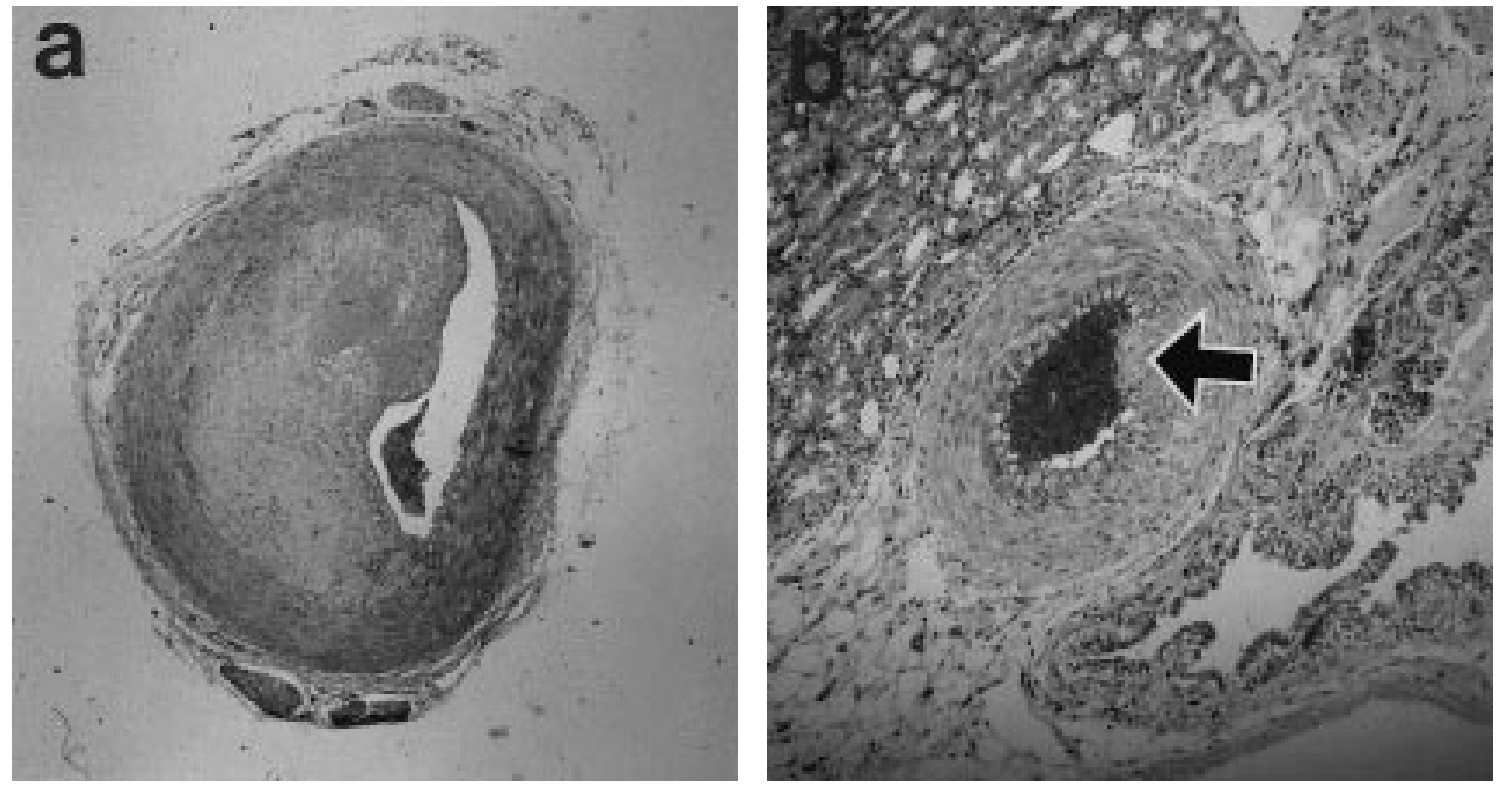

Fig. 2. The influx and intravisceral arteries of the kidney. (a): High-grade luminal stenosis was observed in an influx artery at the hilus renalis $(\times 13)$ (stenotic rate: $82 \%)$. (b): Slight intimal proliferation was recognized in an interlobar artery just behind the hilum of the kidney $(\times 13)$.
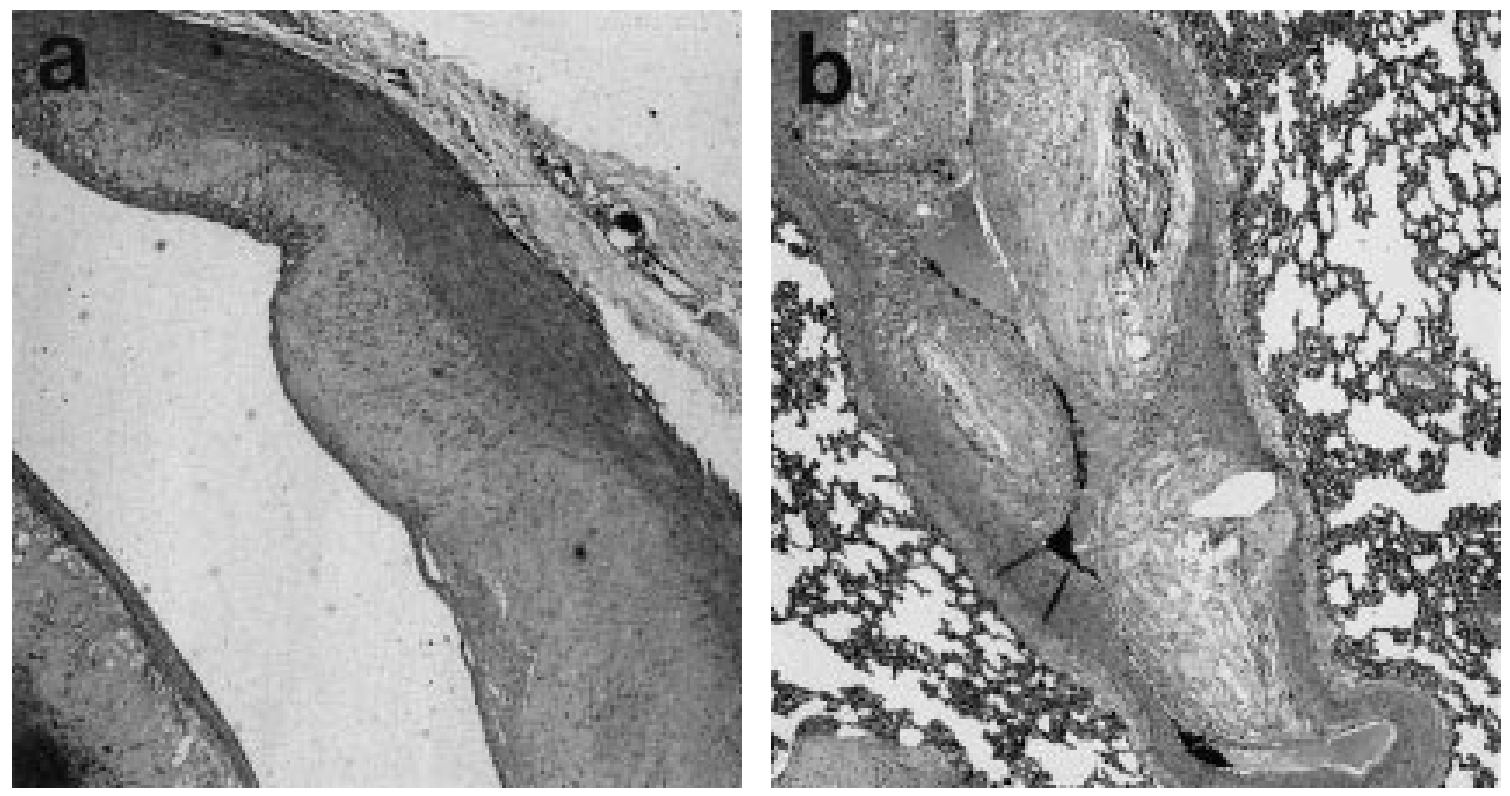

Fig. 3. The influx and intravisceral arteries of the lung. (a): Severe atherosclerotic lesions were recognized in the pulmonary arterial trunk. The lesions were composed of cholesterol clefts, necrosis and fibrous caps $(\times 10)$. (b): Severe atherosclerosis with high-grade lumen stenosis due to the intimal proliferation of foam cells and fibrous connective tissue in a large sized intrapulmonary artery $(\times 10)$.

arterial trunk were atheroma composed of fibrous capsule, cholesterol clefts and necrosis. However, wide hyalinization was characteristic in the hepatic arteries and the main lesions were composed of fibrous capsule in the renal influx arteries.

Furthermore, there were plenty of differences in arte- 
Table 1. Grades of arteriosclerosis

\begin{tabular}{lcc}
\hline Organ & Influx artery & Intravisceral artery \\
\hline Liver & +++ & $-\sim \pm$ \\
Kidney & +++ & $-\sim \pm$ \\
Lung & +++ & $++\sim+++$ \\
\hline
\end{tabular}

-: No lesion. $\pm \sim+$ : Intimal thickening up to $50 \%$ of the perimeter of the lumen. ++ : Concentric arterial lesion with fibrous capsule, cholesterol clefts or necrosis (mild or moderate luminal stenosis estimated at $<50 \%$ ). +++ : Concentric arterial lesion with fibrous capsule, cholesterol clefts or necrosis (severe luminal stenosis estimated at $>50 \%$ ).

riosclerosis formation in the intravisceral arteries in these organs. In the liver and kidney, a distinct arteriosclerotic lesion was never observed in any of the intravisceral arteries, but in the intrapulmonary arteries, high-grade atherosclerotic changes with severe stenosis and complete obstruction of the lumen were recognized.

Napoli [12] reported that the intracranial arteries (middle cerebral and basilar artery) of human fetuses were more resistant to fatty streak formation, due to maternal hypercholesterolemia, than the extracranial (common carotid artery and the abdominal aorta) arteries. He also demonstrated that the activities of superoxide dismutase, catalase and glutathione peroxidase in the intracranial arteries were significantly higher than in the extracranial arteries. This result means that the intracranial arteries have a protective mechanism against free radical-mediated atherogenic processes. It is tempting to speculate that this hypothesis may explain the differences observed in the influx and intravisceral arteries in the liver, kidney and lung, in the present study.

From the consideration of the physiological differences in the brain, liver, kidney and the lung, only the pulmonary perfusion pressures are characteristic. In the lung as with the heart, the perfusion pressure is lower than in the other parenchymatous organ (the liver, kidney and brain) [14]. The low perfusion pressure is a possible cause of atherosclerosis. Furthermore, we cannot ignore the aging factor in arteriosclerotic formation. It is common knowledge that brain atherosclerosis occurs over 10 years later than in the heart. Discrepancies in the formation of arteriosclerosis in the liver, kidney and lung were recognized in the present study. However, now, there is the possibility of future occurrence of arteriosclerosis in the intravisceral arteries of the liver and kidney with aging.

In conclusion, arteriosclerotic changes were observed in all the influx arteries of the liver, kidney and lung, but in the intravisceral arteries of these organs, atherosclerotic lesions with high-grade stenosis or total obstruction of the lumen were recognized only in the lung (Table 1).

From the present study, the lung is an organ predisposed to atherosclerosis, as are the heart and brain, but not the liver and kidney. This result is very interesting because the lung is recognized as an important organ for producing many vasoactive substances and for regulating the systemic circulation.

\section{References}

1. Atkinson, J.B., Hoover, R.L., Berry, K.K., and Swift, L.L. 1989. Atherosclerosis 78: 123-136.

2. Buja, L.M., Clubb, F.J., Bilheimer, D.W., and Willerson, J.T. 1990. Eur. Heart. J. 11: 41-52.

3. Buja, L.M., Kita, T., Goldstein, J.L., Watanabe, Y., and Brown, M.S. 1983. Arteriosclerosis 3: 87-101.

4. Chobanian, A.V. 1990. Clin. Cardiol. 13: 1243-1248.

5. Chobanian, A.V., Lichtenstein, A.H., Nilakhe, V., Haudenschild, C.C., Drago, R., and Nickerson, C. 1989. Hypertension 14: 203-209.

6. Dowell, F.J., Hamilton, C.A., Lindop, G.B., and Reid, J.L. 1995. Arterioscler. Thromb. Vasc. Biol. 15: 1152-1160.

7. Hatanaka, K., Ito, T., Shiomi, M., Yamamoto, A., and Watanabe, Y. 1987. Am. Heart. J. 113: 280-288.

8. Mori, M., Itabe, H., Takatoku, K., Shima, K., Inoue, J., Nishiura, M., Takahashi, H., Ohtake, H., Sato, R., Higashi, Y., Imanaka, T., Ikegami, S., and Takano, T. 1999. J. Biol. Chem. 27; 274: 24828-24837.

9. Mori, M., Iwasaki, K., Sato, R., Komine, Y., Itabe, H., Imanaka, T., and Takano, T. 1996. J. Atheroscler. Thromb. 3: 25-31.

10. Nagano, Y., Nakamura, T., Matsuzawa, Y., Cho, M., Ueda, Y., and Kita, T. 1992. Atherosclerosis 92: 131-140.

11. Nakamura, M., Abe, S., and Kinukawa, N. 1996. Atherosclerosis 124: 37-47.

12. Napoli, C., Witztum, J.L., Nigris, F., Palumbo, G., D’Armient, F.P., and Palinski, W. 1999. Circulation 99: 2003-2010.

13. Nickerson, C.J., Haudenschild, C.C., and Chobanian, A.V. 1992. Exp. Mol. Pathol. 56: 173-185.

14. Ohar, J.A., Pyle, J.A., and Waller, K.S. 1990. Am. Rev. Respir. Dis. 141: 104-110.

15. Shiomi, M., Ito, T., and Watanabe, Y. 1987. Prog. Clin. Biol. Res. 229: 35-40.

16. Watanabe, Y., Ito, T., Shiomi, M., Tsujita, Y., Kuroda, M., Arai, M., Fukami, M., and Tamura, A. 1988. Biochem. Biophys. Acta. 960: 294-302. 